\title{
Docencia en economía de la salud en México: cinco años de experiencia
}

\author{
Teaching of health economics in Mexico: \\ five years of experience
}

\begin{abstract}
Patricia Hernández*, Armando Arredondo*, Carlos Cruz*. Claudia Ortiz*, Luis Durán*, Leticia Avila*
HERNÁNDEZ, P. et al. Docencia en economía de la saiud en México: cinco años de experiencia. $R e v$. Saúde Pública, 28 238-42, 1994. La docencia de una nueva especialidad impone el reto de agilizar la formación de docentes y egresados en cada disciplina. El área de la Economía de la Salud busca responder a los requerimientos de recursos que operen los cambios en los sistemas de salud que se ameritan con el nuevo modelo de desartollo, que sean capaces de generar nuevos conocimientos, preveer y buscar soluciones a las dificultades de los procesos en marcha. Con este fin se elaboró una estrategia de desarrollo integral de la especialidad en México, cuya vertiente de docencia es analizada en este escrito y presentada bajo los siguientes rubros: estructuración teórica de los contenidos; adecuación de los contenidos al alumnado; apoyo a los docentes; intercambio y difusión; y formulación de una agenda de trabajo para el mediano plazo.
\end{abstract}

Descriptores: Economía de la salud. Enseñanza.

\section{Introducción}

El surgimiento de la economía de la salud coincide con una recesión económica en América Latina que conduce actualmente a la modernización de los servicios de salud. Estos cambios se realizan con escaso personal especializado ${ }^{7}$. Asi, la formación de recursos humanos pasa a ser una estrategia fundamental, que a su vez enfrenta la carencia de docentes especializados.

En el caso de México, la dinámica del sistema nacional de salud supone la participación de diversos actores, donde el personal del sector intervenga con creatividad, respaldo técnico y compromiso con los valores de equidad, calidad y eficiencia, principios que se vinculan a la economía de la salud ${ }^{8}$. El papel de los recursos humanos es cuestionar, avanzar y proponer eventuales respuestas a la problemática de la salud pública. Por lo tanto, la responsabilidad de los docentes estriba en asegurar que los egresados, según su nivel de formación, logren participar con su práctica en generar, operar y evaluar políticas, estrategias y programas en los servicios de salud.

La salud pública, como ámbito de acción y desarrollo de ésta nueva especialidad es el marco de operación de un programa docente integral, cuyos avances,

* Instituto Nacional de Salud Pública. Escuela de Salud Pública de México

Separatas/Reprints: P. Hernández - Av. Universidad 655. Cuernevaca Morelos 62508 México con cinco años de experiencia en el país, son presentados en este escrito. La docencia como campo de réplica del conocimiento, requiere de monitoreo y evaluación para identificar los logros y obstáculos en su desempeño. Esto es aún más importante cuando se trata de una especialidad reciente como es la economía de la salud.

\section{Antecedentes del programa docente en eco- nomía de la salud}

La economía de la salud es la especialidad que aplica los principios y técnicas de la economía al campo particular de la salud ${ }^{3}$, siendo la salud diferente de otros bienes en el mercado. Representa una alternativa teórico-metodológica a la problemática existente en los servicios y la necesidad de adaptación del sistema nacional de salud a las nuevas condiciones de la sociedad ${ }^{2}$.

La economía de la salud apoya la toma de decisiones para la asignación y el uso racional de los recursos, permite valorar los costos en función de la efectividad y los beneficios de las distintas alternativas de acción posibles, y facilita una visión integral del proceso de producción, distribución y consumo de bienes y servicios, considerando la oferta, y su contraparte, la demanda, la población, sus necesidades y preferencias ${ }^{6}$. Busca articular los procesos sociales, económicos, políticos, técnicos e institucionales, en el contexto de la salud pública en los distintos niveles de agregación requeridos. La especialidad es aplicable a la dirección y la operación del sistema nacional de salud. 
Por lo tanto se requiere formar personal con diferentes niveles de capacitación técnica que varían desde la información, comprensión, aplicación, adaptación, o la generación teórico-metodológica del conocimiento especializado. El plan docente en economía de la salud pretende homogeneizar y compatibilizar las expectativas de desempeño por los egresados y los requerimientos diferenciados en el manejo de la disciplina en la Escuela de Salud Pública de México (ESPM). Los objetivos de cada nivel de formación son:

a) Informativo y/o de actualización especializada: en los cursos de educación contínua.

b) Aplicación de la información especializada: en especialidades, maestrías en salud pública (en sede y semipresencial), en administración de servicios de salud y la residencia en epidemiología del Instituto Mexicano del Seguro Social (IMSS).

c) Generación de conocimiento, o investigación: maestría en ciencias y doctorado en salud pública.

d) Dirección de servicios de salud y apoyo a la toma de decisiones: se espera que pueda ser realizada por todos los egresados de los programas de maestría y doctorado.

En las escuelas de salud pública del país, ésta materia se incluyó en años recientes. Los docentes de las ciencias sociales participantes del Encuentro de la Asociación Mexicana de Escuelas de Salud Pública de $1992^{\circ}$, refieren que los programas de economía de la salud han carecido de un cuerpo especializado, integral y sistemático. De ahí que este plan docente es el primer programa estructurado de la especialidad en el país.

\section{La estrategia desarrollada}

Este plan docente se formuló con el objetivo de responder a las necesidades de formación de recursos humanos en economía de la salud, e intervino en cuatro distintos planos:

\section{A. Estructuración tebrica de los contenidos.}

Se identificaron las potencialidades de aplicación de ésta especialidad ${ }^{4}$, que cubren cada uno de los momentos del ciclo productivo de los servicios de salud*. Según los niveles de complejidad de cada ámbito, se estructuró una matriz ideal según el área de trabajo ${ }^{1}$ (Tabla), que se ajustó para su aplicación en este plan.

* Incluyen el análisis de los determinantes de la salud; la salud como un bien económico; la demanda, oferta y equilibrio del mercado del complejo médico industrial y los servicios de salud; la evaluación microeconómica de procesos y programas; el financiamiento; la aplicación de los instrumentos de política económica; y la planeación de los servicios.
Se establecieron cinco tipos de curso, según los requerimientos de los programas docentes, que son complementarios. El primer nivel cubre las necesidades de información en sus contenidos (cursos I, II); el segundo es de corte metodológico (cursos III, IV). Un último nivel es flexible, dependiendo del área sustancial y el nivel de los alumnos a quienes esté dirigido $(\mathrm{V})$.

Los cursos se integraron a los programas regulares de formación de recursos humanos en especialidades, maestrías, doctorado en salud pública y de educación contínua. Las unidades didácticas para los programas de postgrado se integraron al sistema de créditos que brinda la posibilidad de asistir a uno o más cursos de economía de la salud, en función del área de especialidad y nivel de formación, que las integra como básicas, u opcionales abiertas a todos los alumnos. Su estructura es la siguiente.

Economía de la salud I: Introducción a la especialidad. Presenta los conceptos de economía, economía de la salud, las nueve áreas de aplicación potenciales (ver Tabla) y su utilidad. Busca informar y apoyar la aplicación de los contenidos, a través de un taller que profundiza en cada tema. Se realiza en $40 \mathrm{~h}$. No tiene prerrequisitos. Se propone que el alumno logre la compresión y uso de la información especializada.

Economía de la salud II: Analiza el proceso de producción de los servicios de salud. Su enfoque es teórico-metodológico y se complementa también con el desarrollo de talleres para cada tema. Se realiza en 40 horas. No tiene prerrequisitos. Se propone la comprensión y uso de la información especializada.

Economía de la salud III: Financiamiento y evaluación económica de la atención médica. El eje central es la evaluación económica de los programas y acciones de salud, y aborda las modalidades de financiamiento de los servicios. Se busca la comprensión, y aplicación de este tipo de análisis por los estudiantes. Se realiza en 40 horas, tiene como prerrequisitos el nivel I. Busca la generación de estos estudios por el alumno.

Economía de la salud IV: Econometría. Introduce a los alumnos a la medición cuantitativa especializada ejercitándose en su aplicación. Busca la generación de conocimiento especializado. Curricularmente es una materia optativa.

Economía de la salud V: Temas selectos de la economía de la salud. Su contenido y nivel es flexible, tanto para programas de formación, como de educación contínua, con objetivos de comprensión o de generación de conocimiento, según el área de formación, trabajo e institución de los participantes. Aprovecha el desarrollo disciplinario en problemas sustantivos de salud y de los servicios, tales como desarrollo y ambiente, gerencia, y tratado de libre comercio. Según la temática y el nivel, cubre de 40 a 120 horas. 
Tabla. Matriz de integración de los diferentes campos de aplicación de la economía de la salud por nivel de formación academica. (Modificado de Arredondo et al'1)(1992).

\begin{tabular}{|c|c|c|c|c|c|c|c|c|}
\hline \multirow{3}{*}{ Campos de aplicación } & \multicolumn{7}{|c|}{ Nivel de formación } & \\
\hline & \multicolumn{4}{|c|}{ Licenciatura } & \multicolumn{4}{|c|}{ Posgrado } \\
\hline & $\mathrm{CSH}$ & CEA & CBS & CFM & $\mathrm{S}$ & SS & Ep & $\mathrm{AH}$ \\
\hline $\begin{array}{l}\text { Determinantes y condicionantes } \\
\text { Qué es salud y su valor } \\
\text { Demanda de atención } \\
\text { Oferta de servicios } \\
\text { Evaluación microeconómica } \\
\text { Equilibrio del mercado } \\
\text { Evaluación de instrumentos de política } \\
\text { Evaluación general del sistema } \\
\text { Planeación, y supervisión }\end{array}$ & $\begin{array}{l}2 \\
2 \\
2 \\
2 \\
1 \\
1 \\
1 \\
1 \\
2\end{array}$ & $\begin{array}{l}2 \\
2 \\
2 \\
2 \\
2 \\
2 \\
1 \\
2 \\
2\end{array}$ & $\begin{array}{l}2 \\
2 \\
2 \\
2 \\
2 \\
1 \\
1 \\
1 \\
2\end{array}$ & $\begin{array}{l}1 \\
1 \\
1 \\
1 \\
2 \\
2 \\
1 \\
1 \\
2\end{array}$ & $\begin{array}{l}5 \\
5 \\
5 \\
5 \\
5 \\
3 \\
5 \\
4 \\
4\end{array}$ & $\begin{array}{l}6 \\
6 \\
6 \\
6 \\
6 \\
5 \\
6 \\
6 \\
6\end{array}$ & $\begin{array}{l}6 \\
5 \\
5 \\
4 \\
4 \\
3 \\
5 \\
4 \\
5\end{array}$ & $\begin{array}{l}3 \\
4 \\
5 \\
5 \\
5 \\
4 \\
5 \\
5 \\
6\end{array}$ \\
\hline $\begin{array}{l}\text { Criterios de análisis: } \\
1=\text { =información general } \\
2=\text { técnicas y procedimientos generales } \\
3=\text { información especializada } \\
4=\text { técnicas y procedimientos especializados } \\
5=\text { análisis y aplicación } \\
6=\text { Innovación }\end{array}$ & \multicolumn{5}{|c|}{$\begin{array}{l}\text { Licenciatura: } \\
\text { CSH=ciencias sociales y humanidades } \\
\text { CEA=ciencias económicos-administrativas } \\
\text { CBS=ciencias biológicas y de la salud } \\
\text { CFM=ciencias físico-matemáticas }\end{array}$} & \multicolumn{3}{|c|}{$\begin{array}{l}\text { Posgrado: } \\
\text { SP=salud pública } \\
\text { SS=sistemas de salud } \\
\text { Ep=epidemiología } \\
\text { AH=administración de hospitales }\end{array}$} \\
\hline
\end{tabular}

\section{B. Adecuación de material didáctico}

Ante las opciones de aplicación de la especialidad, la incipiente tradición de documentación y divulgación de las experiencias y de los avances científicos desarrollados en los sistemas de salud, asi como la dificultad para el acceso y la comprensión a la literatura extranjera, se realizó el doble esfuerzo de compilar y difundir el material especializado y elaborar material didáctico. Ante la heterogeneidad de formación del alumnado, se adecúan los materiales en su complejidad. Las clases y talleres son seguidas de sesiones de preguntas y respuestas por programa de formación. Se complementan con guías de clase, lectura y taller para cada sesión. Existe también, material de autoevaluación para los alumnos.

\section{Apoyo a los docentes}

Se buscó elevar la capacidad docente del profesorado y la formación disciplinaria, a través de seminarios en economía de la salud sobre los distintos tópicos deimportancia. Seanalizóla conceptualización, metodología, aplicación empírica y las necesidades, aplicación y desarrollo del área para cada tema. Esto apoyó la solución de carencias de información en los docentes que proceden de otras especialidades.

Se promovió la interacción con grupos especializados externos, a través del programa de intercambio académico, y un componente de desarrollo docente, tuvo como principal objetivo, agilizar el desarrollo de las capacidades didácticas del profesor y apoyarlo con métodos y técnicas de didáctica y pedagogia, y en la identificación de formas de ejercicio y práctica de los contenidos por los alumnos.

- Lapromoción de la investigación con proyectos para la generación de conocimiento por personal del área, tuvo propósitos teóricos, estratégicos y formativos:

- Teóricos, al cumplir una función generadora, de innovación aplicación y/o actualización del conocimiento en temas prioritarios.

- Estratégicos, al contribuir a formar en los procesos científicos a los profesores-investigadores; sustentar académicamente la docencia; formar y consolidar grupos de trabajo que promueven la colaboración externa.

- Formativos, al incorporar a los alumnos en los proyectos en curso.

Las líneas de investigación que se han desarrollado, cubren los aspectos más relevantes para el país, y las nueve áreas temáticas.

\section{Intercambio académico y difusión.}

El intercambio académico y el desarrollo colaborativo en docencia con otros grupos de trabajo, organismos internacionales, universidades nacionales y extranjeras, y centros de servicio, investigación y docencia en economía de la salud, de tipo público y privado, buscó complementar la formación y promover la discusión académica de los docentes, con otros grupos de trabajo. Las modalidades concretas del intercambio consisten en visitas de campo, discusión de estratégias docentes y contenidos, intercambio de material docente e informativo, cursos internacionales con participación de maestros invitados, dotación de becas, asesoría y mayor presencia de los docentes en eventos científicos. 


\section{Agenda de trabajo futuro}

Los avances alcanzados durante este período, han aprovechado la experiencia y colaboración de otros países, principalmente europeos. Creemos que esta apertura requiere también de ser extendida hacia países de la región, en términos de potencialidades de formación conjunta, colaboración, y de compartir enfoques, experiencias y perspectivas. Este sería el camino para establecer una agenda de trabajo que responda más a las necesidades y expectativas de salud y la formación de especialistas en América Latina, y que de acuerdo con la experiencia en México, pudiera cubrir los seguientes puntos:

- Recursos humanos. Consolidar una planta docente en economía de la salud y sus subsespecialidades, que asegure mayor profundidad del manejo de los contenidos.

- Recursos financieros. Incremento de recursos para el apoyo de programas de formación docente, investigación, y becas para los alumnos, que faciliten su concentración en el área.

- Aspectos técnicos. Ampliar la articulación de los contenidos, con las necesidades de formación especializada en el sector, los alumnos de los programas, y las necesidades de salud de la población.

- Estrategias docentes. Apoyar la adquisición de habilidades teórico-prácticas en los alumnos, según requerimientos. La estrategia incluye la generación de un banco de ejercicios, información y material de clase. - Ampliar la cobertura y contenidos de los cursos de educación contínua en niveles, temas y tecnologia para cubrir los requerimientos emergentes y apoyar la resolución de problemas concretos en el sector.

- Vinculación docencia-investigación. Consolidar la línea de investigación y enriquecer las unidades didácticas con los resultados de la investigación en el área. - Vinculación con los servicios. Sistematizar la interacción con los grupos de población, el sistema de atención y los decisores diversos para uso de los hallazgos de investigación, y desarrollo de tesis.

- Iniciar un programa de maestría en economía de la salud, para consolidar los avances en docencia alcanzados.

- Interacción regional de especialistas para compartir y aprovechar las experiencias en forma ágil.

- Establecer un programa de seguimiento, apoyo e interacción con alumnos egresados a través de asociaciones gremiales.

Con estas acciones se espera enriquecer la formación de especialistas que puedan participar en la solución de la problemática de salud en la región.

\section{Conclusiones}

Hasta hoy, gran parte de los esfuerzos desarrollados han sido promisorios para promover la docencia de la disciplina. Se implantó la especialidad dentro de los programas académicos de la Escuela de Salud Pública de México. Sin embargo, la integración teórico-práctica de los contenidos debe promoverse y evaluar los alcances a través del seguimiento de los logros en investigación y la experiencia de los alumnos egresados.

A lo largo de estos cinco años, el perfil de los alumnos ha variado, de ser inicalmente tomadores de decisiones, a otro más variado en su formación y ubicación laboral, con predominio de mandos medios $^{5}$. Esto ha obligado a modificar el esfuerzo informativo y promocional en los cursos iniciales, a un enfoque más metodologico y aplicativo, que responda a las expectativas de los alumnos y las necesidades de los servicios.

Tanto la formación docente como la disciplinaria, jugaron un papel importante en la ampliación progresiva de la planta docente y los programas. El apoyo a la investigación ha mostrado su éxito con la incorporación de elementos de economía de la salud en las tesis, o estar éstas completamente orientadas a la especialidad.

Sin embargo, se amerita realizar una evaluación, establecida no solamente con indicadores de proceso, sino valorando la contribución en la solución de los problemas del sector salud por los egresados. Este ha sido desde un inicio el principal objetivo para desarrollar la especialidad en cuestión.

La aplicación de conocimientos pasa también por asegurar que el trabajo en esta especialidad, conserve su sentido integral y multidisciplinario, y sa una herramienta de intervención en los sistemas de salud.

En la región existen muchos problemas, experiencias y alternativas comunes que pueden ser compartidas. Creemos que los avances en la formación de recursos especializados en Economía de la Salud es un claro ejemplo de ello.

HERNÁNDEZ, P. et al. [Teaching of health economics in Mexico: five years of experience]. Rev. Saúde Pública, 28: $238-42,1994$. Teaching strategies for a new specialty constitute a challenge to ensure the rapid development of human resources for the purpose of supporting these activities. In the health economics field a plan was applied for coping with the needs of teaching activities in the field. The activities developed in the plan are presented in this paper, included under the following items: A. Theoretical organization of the contents of the subject. B. Organization of contents according to the students' needs. C. Support given to teachers. D. The whole strategy also includes: academic interchange, technical advisory services, dissemination of results through meetings, publications and academic organizations. E. Median term agenda: Human resources; Financial resources; Teaching strategies; Interaction between health services and decision makers; and Collabortive network.

Keywords: Health economics. Teaching. 


\section{Referencias Bibliográficas}

1. ARREDONDO, A.; CRUZ, C.; HERNÁNDEZ, P. México: la formación de recursos humanos en economía de la salud. Educ. Méd. Salud, 25: 254-67, 1991.

2. CRUZ, C. \& HERNÁNDEZ, P. Economía de la salud: reflexiones en materia educativa. J. Health Adm. Educ., 9: 361-93, 1991.

3. DRUMMOND, M. Principios de evaluación econónica en asistencia sanitaria. Madrid, Instituto de Estudios Laborales y de Seguridad Social, 1983.

4. FERREIRA, J.R. El liderazgo en salud pública en América. Educ. Méd. Salud, 23: 5-13, 1989.

5. HERNÁNDEZ, P.; AVILA, L.; ORTIZ, C.; RODRIGUEZ, G. La economía de la salud en la modemización de los servicios. In: Congreso Mundial de Medicina Social, Guadalajara/ Jalisco, 1994
6. MILLS, A. \& GILSON, L. Health economics for developing countries: a survival kit. London, Survival Kit, 1988. (EPC Publication, 17).

7. MUSGROVE, P. Introducción. In: Organización Panamericana de la Salud. Economía de la salud: perspectivas para América Latina. Washington, D.C., 1989. (OPAS - Publicación Científica, 517).

8. PROGRAMA Nacional de Salud. 1990-1994. Parte II. Objetivo General, políticas y estratégias. México, 1990.

9. REUNIÓN ANUAL DE LA ASOCIACIÓN MEXICANA DE ESCUELAS DE SALUD PÚBLICA, Cuernavaca, 1992.

Recebido para publicação em 9.11 .1993

Reapresentado em 5.4.1994 Aprovado para publicaçẫo em 19.5.1994 